\title{
A convenient and general procedure for the synthesis of $\alpha$-ureidophosphonates under catalyst-free conditions
}

\author{
Babak Kaboudin, ${ }^{* a}$ Mohammad Bagher Afsharinezhad, ${ }^{a}$ and Tsutomu Yokomatsu ${ }^{b}$ \\ ${ }^{a}$ Department of Chemistry, Institute for Advanced Studies in Basic Sciences (IASBS), \\ Gava Zang, Zanjan 45137-66731, Iran \\ ${ }^{b}$ School of Pharmacy, Tokyo University of Pharmacy and Life Sciences, \\ 1432-1 Horonouchi, Hachioji, Tokyo 192-0392, Japan \\ E-mail: kaboudin@iasbs.ac.ir
}

\section{Dedicated to Prof. Pawel Kafarski to honor the achievements within his career}

\begin{abstract}
We report here a novel and convenient method for the synthesis of $\alpha$-ureidophosphonates through a one-pot reaction of three component condensation of aldehyde with urea and diethylphosphite under catalyst-free conditions in toluene at reflux. Treatment of aldeyhde with a mixture of urea and diethylphosphite gives a $\alpha$-ureidophosphonate in moderate yield. The $\alpha$ ureidophosphonate product was easily separated and crystallized from the reaction mixture.
\end{abstract}

Keywords: Aminophosphonates, $\alpha$-ureidophosphonates, urea, aldehyde, condensation reaction, catalyst-free

\section{Introduction}

The organic chemistry of phosphorus compounds have became increasingly useful and important in organic synthesis. Among the organophosphorus compounds, phosphonic acids are important class of compounds that exhibit a variety of interesting and useful properties. ${ }^{1}$ The synthesis of $\alpha$-substituted phosphoryl derivatives (phosphonic and phosphinic acids) has attracted significant attention due to their biological activities with broad application as enzyme inhibitors, antimetabolites and antibiotics. ${ }^{2}$ Among $\alpha$-functionalized phosphonic acids, $\alpha$ aminoalkylphosphonic derivatives have biological activities such as anti-bacterial, ${ }^{3}$ herbicidal ${ }^{4}$ and fungicidal. ${ }^{5}$ Aminoalkylphosphonic acids, the phosphonic acid analogues of 1-amino carboxylic acids, are important class of compounds that exhibit a variety of interesting and useful properties. ${ }^{6}$ While the synthesis and properties of many types of aminophosphonic acid derivatives have been widely investigated, ${ }^{7-9}$ studies of $\alpha$-ureidophosphonates have received little 
attention. $\alpha$-Ureidophosphonates have been used as precursor for the synthesis of chiral 1aminophosphonates. ${ }^{10} \alpha$-Ureidophosphonates have shown moderate activity against number of fungal pathogens. ${ }^{11}$ They also display powerful antiviral activities against TMV. ${ }^{12}$ Besides their biological importance, $\alpha$-ureidophosphonates are also known for their metal chelating ability. ${ }^{13}$ In addition, these compounds used as active ingredients in pesticides especially insecticides and acaricides. $^{14}$

In contrast to extensive studies on the synthesis of 1-aminophosphonic acid derivatives, ${ }^{15}$ relatively few papers have been reported on the synthesis of $\alpha$-ureidophosphonates. Synthetic routes to 1-ureidophosphonates involve prolonged heating of three component reaction of trialkylphosphite with urea and aldehydes at $50{ }^{\circ} \mathrm{C}$ in the presence of $\mathrm{BF}_{3}$ as a catalyst under nitrogen described by Birum, ${ }^{16}$ and the reaction of aminophosphonates with isocyanates in the presence of LDA at $-30^{\circ} \mathrm{C} .{ }^{17}$ However there are some problems associated with these methods including harsh reaction conditions, long reaction times and side reactions. On the other hand, three component reaction of trialkylphosphite with urea and aldehydes at $50{ }^{\circ} \mathrm{C}$ in the presence of $\mathrm{BF}_{3}$ as a catalyst under nitrogen, gave urylenediphosphonates as major product. In this method the formation of urea monophosphonates ( $\alpha$-ureidophosphonates) is accompanied by the formation of urylenediphosphonates, even when equimolar quantities of reactants was used and pure $\alpha$-ureidophosphonates are difficult to isolate. As part of our efforts to explore the novel methods for the synthesis of organophosphorus compounds, ${ }^{18}$ we report a new method for the synthesis of $\alpha$-ureidophosphonates from the one-pot three component condensation of aldehyde with urea and diethylphosphite under catalyst-free conditions in toluene at reflux.

\section{Results and Discussion}

Treatment of $p$-chlorobenzaldehyde (1a), as a model compound, with a mixture of urea and diethylphosphite was studied in toluene, and the progress of the reaction was monitored by TLC. Treatment of 1a with urea in the presence of diethylphosphite in dry toluene at room temperature failed after $24 \mathrm{~h}$ to form any product. When the reaction was carried out at reflux for $7 \mathrm{~h}$, a mixture of products was obtained (Scheme 1). The ${ }^{31} \mathrm{P}-\mathrm{NMR}$ spectrum of this mixture exhibited three peaks at $\delta 22.53,22.82$ and $23.11 \mathrm{ppm}$ due to the mixture of two diastereoisomers $3 \mathbf{a}$ (in a 63:37 ratio of diastereoisomers, Scheme 1) and diethyl $\alpha$-ureido(4-chlorophenyl)methylphosphonate $\mathbf{2 a}$.

When the reaction mixture was subjected to washing with water, and evaporation of the solvent, only $\mathbf{2 a}$ crystallized from a mixture of diethyl ether and $n$-hexane. The structure of $\mathbf{2 a}$ was confirmed by NMR data and X-ray analysis. In the ${ }^{31} \mathrm{P}-\mathrm{NMR}$ spectrum, a singlet peak appeared at $\delta 22.50 \mathrm{ppm}$. The ${ }^{1} \mathrm{H}-\mathrm{NMR}$ spectrum had a doublet of doublets peak at $5.40 \mathrm{ppm}$

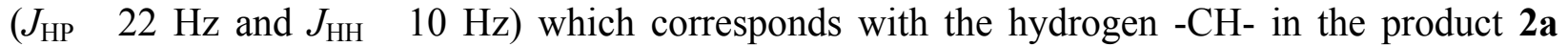
( simplified from doublet of doublets to doublet by addition of $\mathrm{D}_{2} \mathrm{O}$ ). The ${ }^{13} \mathrm{C}$-NMR spectrum exhibited doublet peak at $\delta 50.2\left(J_{\mathrm{CP}} 152 \mathrm{~Hz}\right)$. 
<smiles>CCOC(=O)C(NC(N)=O)c1ccc(Cl)cc1</smiles><smiles>CCOP(=O)(OCC)[C@H](NC(=O)NC(c1ccc(Cl)cc1)c1ccc(Cl)cc1)P(=O)=O</smiles><smiles>CCOC(=O)C(NC(=O)N[C@H](C(=O)OCC)c1ccc(Cl)cc1)P(=O)=O</smiles>

2a

3a

\section{Scheme 1}

The structure was supported by its X-ray analysis. We could obtain suitable crystals for 2 a for X-ray analysis. The crystal structure of $\mathbf{2 a}$ is illustrated in Figure 1.

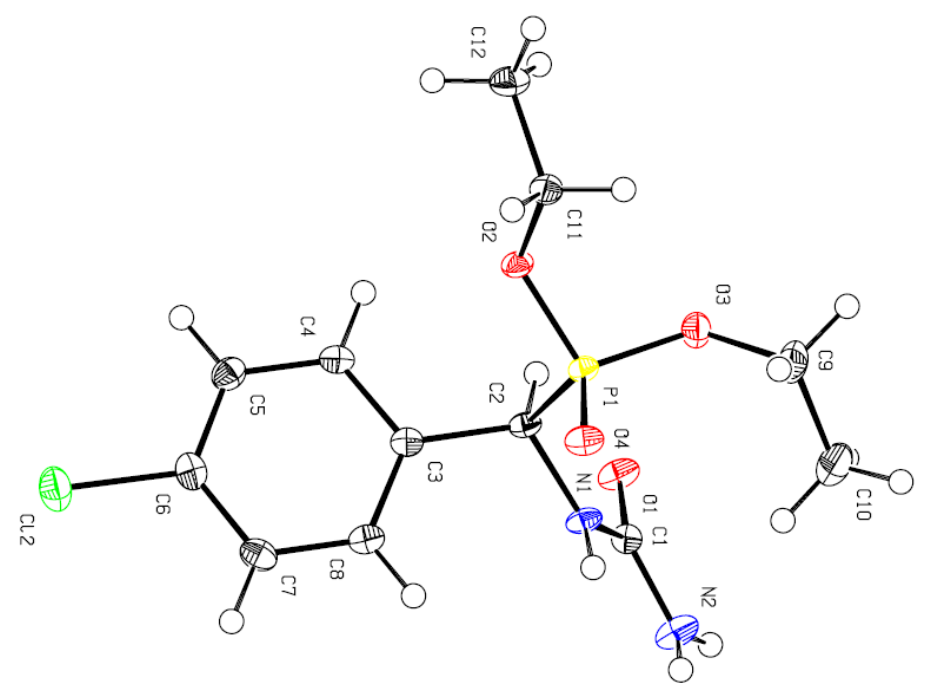

Figure 1. ORTEP drawing of $\mathbf{2 a}$.

This process was successfully applied to other aldehydes $\mathbf{1}$ as summarized in Table 1 . As shown in Table 1, the reaction of different substituted (ortho, meta and para)- of benzaldehydes with a mixture of urea and diethylphosphite in dry toluene at reflux, afforded the corresponding $\alpha$-ureidophosphonates derivatives 2 in 30-54\% isolated yields (entries 1-10). Treatment of benzaldehyde and thiophene-2-carbaldehyde with a mixture of urea and diethylphosphite in toluene at reflux, gave corresponding urylenediphosphonate $\mathbf{3 k}$ and $\mathbf{3} \mathbf{I}$ as major product (entries 11 and 12). 
Table 1. Synthesis of $\alpha$-ureidophosphonates via one-pot three component condensation of aldehyde, urea and diethylphosphite

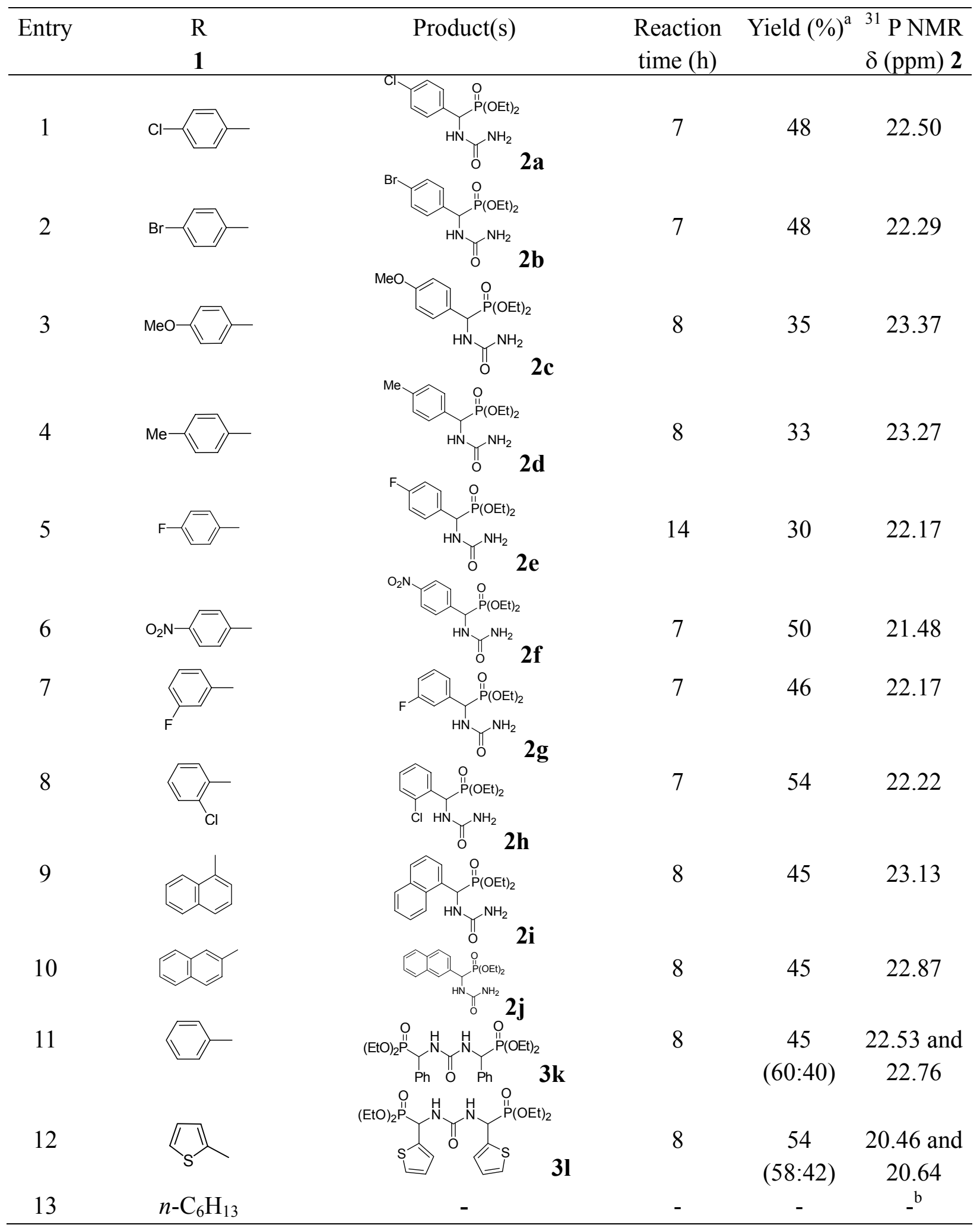

${ }^{\mathrm{a}}$ Yields refer to the isolated pure products. ${ }^{\mathrm{b}}$ Unknown mixture. 
When the reaction mixture of benzaldehyde and thiophene-2-carbaldehyde was subjected to washing with water followed by adding a mixture of solvents diethyl ether and $n$-hexane, corresponding $\alpha$-ureidophosphonates (2k and $\mathbf{2 l}$ ) did not crystallize from the reaction mixture. The reaction of heptanal, as an aliphatic aldehyde, with a mixture of urea and diethylphosphite in toluene at reflux, afforded an unknown mixture (entry 13).

Birum reported three component reaction of trialkylphosphite with urea and aldehyde at 50 ${ }^{\circ} \mathrm{C}$ in the presence of $\mathrm{BF}_{3}$ as a catalyst under nitrogen, but this method gave urylenediphosphonates as major product. ${ }^{16 a}$ In this catalytic method, three component reaction of trialkylphosphite with thiourea and aldehydes at $50{ }^{\circ} \mathrm{C}$ in the presence of $\mathrm{BF}_{3}$ as a catalyst under nitrogen, failed to give the corresponding adduct. ${ }^{16 \mathrm{a}} \mathrm{We}$ were interested to examine the three component reaction of dialkylphosphite with thiourea and 4-chlorobenzaldehyde (1a) in toluene at reflux. Treatment of 1a with thiourea in the presence of diethylphosphite in dry toluene at room temperature failed after $24 \mathrm{~h}$ to form any product. When the reaction was carried out at reflux for $7 \mathrm{~h}$, gave corresponding $\alpha$-thioureidophosphonate $4 \mathrm{a}$ in $48 \%$ isolated yield (Scheme 2).

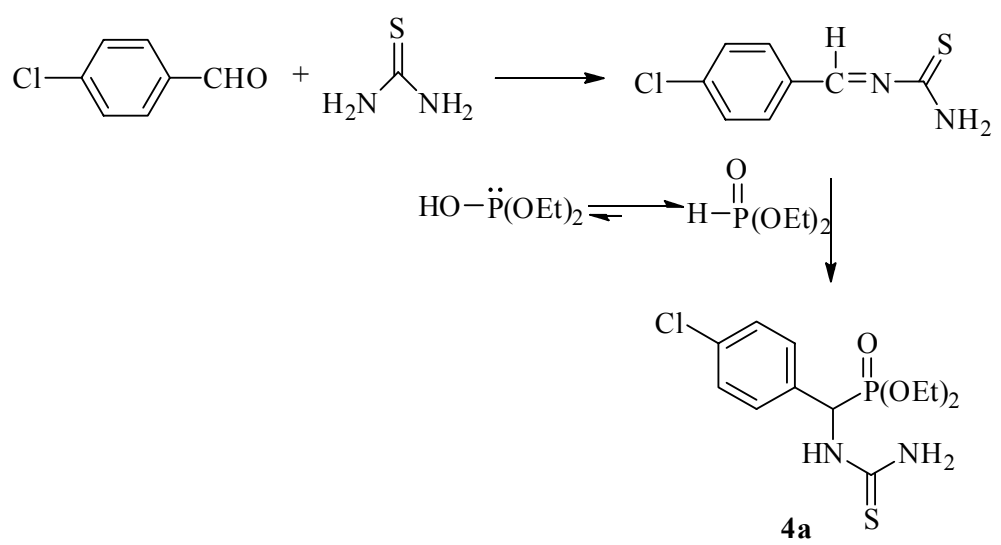

\section{Scheme 2}

\section{Conclusions}

We have developed a simple and practical approach for the synthesis of $\alpha$-ureidophosphonates. Through a three component reaction of aldehydes with a mixture of urea and diethylphosphite under catalyst-free conditions in toluene at reflux, $\alpha$-ureidophosphonates can be synthesized cleanly in moderate yield. The structure of $\alpha$-ureidophosphonates was determined by NMR data and X-ray analysis. ${ }^{20}$ Treatment of aldehydes with thiourea in the presence of diethylphosphite in dry toluene at reflux for $7 \mathrm{~h}$, gave corresponding $\alpha$-thioureidophosphonate in moderate isolated yield. Some of the major advantages of this protocol are: simple procedure, easy work-up, and catalyst-free conditions. 


\section{Experimental Section}

General. All chemicals were commercial products and distilled or recrystallized before use. NMR spectra were taken with a $400 \mathrm{MHz}$ Brucker Avance instrument with the chemical shifts being reported as $\delta$ ppm and couplings expressed in Hertz. Silica gel column chromatography was carried out with Silica gel 100 (Merck No. 10184). Merck Silica-gel 60 F254 plates (No. 5744) were used for the preparative TLC. Mass spectra were measured on a LCMASS micromass LCT and Micromass Autospec. Melting points are uncorrected.

X-Ray crystal data of $\mathbf{2 a}$ were collected by a diffractometer. The structure was solved by a direct method using SHLEXS-97 (Scheldrik, 1997) and refined with a full matrix laser-squares method. Molecular formula $\mathrm{C}_{12} \mathrm{H}_{18} \mathrm{ClN}_{2} \mathrm{O}_{4} \mathrm{P}$, MW 320.07, Monoclinic, space group Pna21/c, a = 7.8880(11) $\AA, \mathrm{b}=25.221(3) \AA, \mathrm{c}=9.1789(9) \AA, \mathrm{V}=1515.4(3) \AA 3, \mathrm{~T}=90 \mathrm{~K}, \mathrm{Z}=4, \mathrm{Dx}=1.406$ $\mathrm{Mg} / \mathrm{m} 3, \quad(\mathrm{Mo}-\mathrm{K} \alpha)=0.71073 \AA$, absolute structure parameter $=0.12(10), \quad \mathrm{R}=0.0193$ over independent reflections (3451). Crystallographic data (excluding structure factors) for the X-ray crystal structure analysis reported in this paper have been deposited with the Cambridge Crystallographic Data Center (CCDC) as supplementary publication No. CCDC 835018, copies of these data can be obtained, free of charge, upon application to CCDC, 12 Union Road, Cambridge CB2 1EZ, UK [fax:+44(0)-1223-336033 or e-mail: deposit@ccdc.cam.ac.uk].

Synthesis of $\boldsymbol{\alpha}$-ureidophosphonates (2). The aldehyde $(10 \mathrm{mmol})$ was added to a stirred mixture of urea $(1 \mathrm{~g}, 3.07 \mathrm{mmol})$ and diethylphosphite $(10 \mathrm{mmol})$ in dry toluene $(50 \mathrm{~mL})$ at reflux and the solution was stirred for 7-14 h. Stirring was continued for $1 \mathrm{~h}$ at room temperature and then the reaction mixture was washed with water $(3 \times 50 \mathrm{~mL})$ and the solvent was removed under vacuum. A mixture of 50:50 diethyl ether $n$-hexane $(50 \mathrm{~mL})$ was added to the reaction mixture and pure product 2 was crystallized from the mixture. All products gave satisfactory spectral data in accord with the assigned structures.

Diethyl [a-ureido-(4-chlorophenyl)]methyl phosphonate (2a). White solid, mp 202-204 ${ }^{\circ} \mathrm{C}$; ${ }^{1} \mathrm{H}$ NMR $\left(\mathrm{CDCl}_{3}, 400 \mathrm{MHz}\right), \delta: 1.15(\mathrm{t}, J 7.2 \mathrm{~Hz}, 3 \mathrm{H}), 1.40(\mathrm{t}, J 7.2 \mathrm{~Hz}, 3 \mathrm{H}), 3.67-3.81(\mathrm{~m}, 1 \mathrm{H})$, 3.86-3.98 (m, 1H), 4.16-4.32 (m, 2H), 5.35 (br, $\left.\mathrm{NH}_{2}, 2 \mathrm{H}\right), 5.39$ (dd, J $\left.22.0 \mathrm{~Hz}, J 10.0 \mathrm{~Hz}, 1 \mathrm{H}\right)$, 7.32 (d, $J 8.0 \mathrm{~Hz}, 2 \mathrm{H}), 7.41$ (dd, J $8.4 \mathrm{~Hz}, J 2.0 \mathrm{~Hz}, 2 \mathrm{H}), 7.65$ (d, $J 3.2 \mathrm{~Hz}, 1 \mathrm{H}, \mathrm{NH}) .{ }^{13} \mathrm{C}$ NMR $\left(\mathrm{CDCl}_{3}-\mathrm{TMS}, 100.6 \mathrm{MHz}\right), \delta: 16.2\left(\mathrm{~d}, J_{\mathrm{PC}} 6.0 \mathrm{~Hz}\right), 16.2\left(\mathrm{~d}, J_{\mathrm{PC}} 6.0 \mathrm{~Hz}\right), 50.1$ (d, $\left.J_{\mathrm{PC}} 155.0 \mathrm{~Hz}\right)$, $63.5\left(\mathrm{~d}, J_{\mathrm{PC}} 7.0 \mathrm{~Hz}\right), 63.9\left(\mathrm{~d}, J_{\mathrm{PC}} 7.0 \mathrm{~Hz}\right), 128.7$ (d, $\left.J_{\mathrm{PC}} 2.0 \mathrm{~Hz} \mathrm{~Hz}\right), 129.4$ (d, $\left.J_{\mathrm{PC}} 6.0 \mathrm{~Hz}\right), 133.9$ $\left(\mathrm{d}, J_{\mathrm{PC}} 3.0 \mathrm{~Hz}\right), 134.6,158.4\left(\mathrm{~d}, J_{\mathrm{PC}} 10.0 \mathrm{~Hz}\right) .{ }^{31} \mathrm{P} \mathrm{NMR}\left(\mathrm{CDCl}_{3} / \mathrm{H}_{3} \mathrm{PO}_{4}\right), \delta: 22.50$. EI-MS: $\mathrm{m} / z$ $320\left(\mathrm{M}^{+}\right)$.

Diethyl [a-ureido-(4-bromophenyl)]methyl phosphonate (2b). White solid, mp 197-199 ${ }^{\circ} \mathrm{C} .{ }^{1} \mathrm{H}$ NMR (DMSO, $400 \mathrm{MHz}), \delta: 1.10(\mathrm{t}, J 7.2 \mathrm{~Hz}, 3 \mathrm{H}), 1.21$ (t, $J 7.2 \mathrm{~Hz}, 3 \mathrm{H}), 3.76-3.86(\mathrm{~m}, 1 \mathrm{H})$, 3.86-3.96 (m, 1H), 3.97-4.10 (m, 2H), $5.16(\mathrm{dd}, J 22.4 \mathrm{~Hz}, J 9.6 \mathrm{~Hz}, 1 \mathrm{H}), 7.10$ (dd, J 9.6 Hz, $J$ $4.0 \mathrm{~Hz}, 1 \mathrm{H}, \mathrm{NH}), 7.31(\mathrm{dd}, J 8.2 \mathrm{~Hz}, J 2.0 \mathrm{~Hz}, 2 \mathrm{H}), 7.52(\mathrm{~d}, J 8.2 \mathrm{~Hz}, 2 \mathrm{H}) .{ }^{13} \mathrm{C} \mathrm{NMR}\left(\mathrm{CDCl}_{3}\right.$, 100.6 MHz), $\delta: 16.5\left(\mathrm{~d}, J_{\mathrm{PC}} 6.0 \mathrm{~Hz}\right), 16.7\left(\mathrm{~d}, J_{\mathrm{PC}} 6.0 \mathrm{~Hz}\right), 50.1\left(\mathrm{~d}, J_{\mathrm{PC}} 152.0 \mathrm{~Hz}\right), 62.7\left(\mathrm{~d}, J_{\mathrm{PC}} 7.0\right.$ $\mathrm{Hz}), 63.1$ (d, $\left.J_{\mathrm{PC}} 7.0 \mathrm{~Hz}\right), 121.0\left(\mathrm{~d}, J_{\mathrm{PC}} 3.0 \mathrm{~Hz} \mathrm{~Hz}\right), 130.4$ (d, $\left.J_{\mathrm{PC}} 5.0 \mathrm{~Hz}\right), 131.5$ (d, $\left.J_{\mathrm{PC}} 2.0 \mathrm{~Hz}\right)$, 
137.2, 157.9 (d, $\left.J_{\mathrm{PC}} 10.0 \mathrm{~Hz}\right) .{ }^{31} \mathrm{P} \mathrm{NMR}\left(\mathrm{CDCl}_{3} / \mathrm{H}_{3} \mathrm{PO}_{4}\right), \delta: 22.29$. Anal. Calcd for $\mathrm{C}_{12} \mathrm{H}_{18} \mathrm{BrN}_{2} \mathrm{O}_{4}$ P. C, 39.56; H, 4.94; N, 7.69. Found: C, 39.40; H, 4.91; N, 7.53.

Diethyl [ $\alpha$-ureido-(4-methoxyphenyl)]methyl phosphonate (2c). White solid, mp196-198 ${ }^{\circ} \mathrm{C}$. ${ }^{1} \mathrm{H}$ NMR $\left(\mathrm{CDCl}_{3}, 400 \mathrm{MHz}\right), \delta: 1.11(\mathrm{t}, J 6.4 \mathrm{~Hz}, 3 \mathrm{H}), 1.39(\mathrm{t}, J 6.4 \mathrm{~Hz}, 3 \mathrm{H}), 3.62-3.73(\mathrm{~m}, 1 \mathrm{H})$, $3.82(\mathrm{~s}, 3 \mathrm{H}), 3.83-3.94(\mathrm{~m}, 1 \mathrm{H}), 4.12-4.30(\mathrm{~m}, 2 \mathrm{H}), 5.28\left(\mathrm{br}, \mathrm{NH}_{2}, 2 \mathrm{H}\right), 5.34$ (dd, J 21.2 Hz, J 8.4 $\mathrm{Hz}, 1 \mathrm{H}), 6.88$ (d, J 7.6 Hz, 2H), 7.41 (d, J 7.6 Hz, 2H), 7.59 (br, NH, 1H). ${ }^{13} \mathrm{C} \mathrm{NMR}\left(\mathrm{CDCl}_{3}\right.$, 100.6 MHz), $\delta: 16.2\left(\mathrm{~d}, J_{\mathrm{PC}} 7.0 \mathrm{~Hz}\right), 16.5\left(\mathrm{~d}, J_{\mathrm{PC}} 6.0 \mathrm{~Hz}\right), 49.9\left(\mathrm{~d}, J_{\mathrm{PC}} 155.0 \mathrm{~Hz}\right), 55.3,63.3(\mathrm{~d}$, $\left.J_{\mathrm{PC}} 8.0 \mathrm{~Hz}\right), 63.6\left(\mathrm{~d}, J_{\mathrm{PC}} 7.0 \mathrm{~Hz}\right), 113.9,128.0,129.3\left(\mathrm{~d}, J_{\mathrm{PC}} 7.0 \mathrm{~Hz} \mathrm{~Hz}\right), 158.8$ (d, $\left.J_{\mathrm{PC}} 10.0 \mathrm{~Hz}\right)$, 159.3. ${ }^{31} \mathrm{P} \mathrm{NMR}\left(\mathrm{CDCl}_{3} / \mathrm{H}_{3} \mathrm{PO}_{4}\right), \delta$ : 23.37. Anal. Calcd for $\mathrm{C}_{13} \mathrm{H}_{21} \mathrm{~N}_{2} \mathrm{O}_{5} \mathrm{P} . \mathrm{C}, 49.35 ; \mathrm{H}, 6.69 ; \mathrm{N}$, 8.86. Found: C, 47.20; H, 6.82; N, 9.10.

Diethyl [a-ureido-(4-methylphenyl)]methyl phosphonate (2d). White solid, mp 188-190 ${ }^{\circ} \mathrm{C}$. ${ }^{1} \mathrm{H} \mathrm{NMR}\left(\mathrm{CDCl}_{3}, 400 \mathrm{MHz}\right), \delta: 1.11(\mathrm{t}, J 7.2 \mathrm{~Hz}, 3 \mathrm{H}), 1.39(\mathrm{t}, J 7.2 \mathrm{~Hz}, 3 \mathrm{H}), 2.35(\mathrm{~s}, 3 \mathrm{H}), 3.62-$ $3.74(\mathrm{~m}, 1 \mathrm{H}), 3.83-3.95(\mathrm{~m}, 1 \mathrm{H}), 4.17-4.32(\mathrm{~m}, 2 \mathrm{H}), 5.30-5.40\left(\mathrm{br}, \mathrm{NH}_{2}, 2 \mathrm{H}\right), 5.38$ (dd, J 21.6 $\mathrm{Hz}, J 9.6 \mathrm{~Hz}, 1 \mathrm{H}), 7.16(\mathrm{~d}, J 7.8 \mathrm{~Hz}, 2 \mathrm{H}), 7.38(\mathrm{~d}, J 7.8 \mathrm{~Hz}, 2 \mathrm{H}), 7.65(\mathrm{~s}, \mathrm{NH}, 1 \mathrm{H}) .{ }^{13} \mathrm{C}$ NMR $\left(\mathrm{CDCl}_{3}, 100.6 \mathrm{MHz}\right), \delta: 16.1\left(\mathrm{~d}, J_{\mathrm{PC}} 6.0 \mathrm{~Hz}\right), 16.5\left(\mathrm{~d}, J_{\mathrm{PC}} 6.0 \mathrm{~Hz}\right), 21.2,50.4\left(\mathrm{~d}, J_{\mathrm{PC}} 156.0 \mathrm{~Hz}\right)$, $63.4\left(\mathrm{~d}, J_{\mathrm{PC}} 7.0 \mathrm{~Hz}\right), 63.6\left(\mathrm{~d}, J_{\mathrm{PC}} 7.0 \mathrm{~Hz}\right), 128.0$ (d, $\left.J_{\mathrm{PC}} 6.0 \mathrm{~Hz} \mathrm{~Hz}\right), 129.2$ (d, $\left.J_{\mathrm{PC}} 2.0 \mathrm{~Hz}\right), 132.8$, $137.6\left(\mathrm{~d}, J_{\mathrm{PC}} 2.0 \mathrm{~Hz}\right), 158.6\left(\mathrm{~d}, J_{\mathrm{PC}} 10.0 \mathrm{~Hz}\right) .{ }^{31} \mathrm{P} \mathrm{NMR}\left(\mathrm{CDCl}_{3} / \mathrm{H}_{3} \mathrm{PO}_{4}\right), \delta: 23.27$. Anal. Calcd for $\mathrm{C}_{13} \mathrm{H}_{21} \mathrm{~N}_{2} \mathrm{O}_{4}$ P. C, 51.98; H, 7.05; N, 9.33. Found: C, 51.76; H, 7.0 ; N, 9.43.

Diethyl [a-ureido-(4-flourophenyl)]methyl phosphonate (2e). White solid, mp198-200 ${ }^{\circ} \mathrm{C} .{ }^{1} \mathrm{H}$ NMR $\left(\mathrm{CDCl}_{3}, 400 \mathrm{MHz}\right), \delta: 1.09$ (t, $\left.J 7.2 \mathrm{~Hz}, 3 \mathrm{H}\right), 1.13$ (t, $\left.J 7.2 \mathrm{~Hz}, 3 \mathrm{H}\right), 4.78-4.90(\mathrm{~m}, 1 \mathrm{H})$, 4.93-5.12 (m, 3H), $5.38(\mathrm{dd}, J 21.6 \mathrm{~Hz}, J 10.0 \mathrm{~Hz}, 1 \mathrm{H}), 6.97$ (t, $J 8.4 \mathrm{~Hz}, 2 \mathrm{H}), 7.33\left(\mathrm{br}, \mathrm{NH}_{2}\right.$, 2H), 7.35-7.42 (m, 2H). ${ }^{13} \mathrm{C} \mathrm{NMR}\left(\mathrm{CDCl}_{3}, 100.6 \mathrm{MHz}\right), \delta: 16.1$ (d, $\left.J_{\mathrm{PC}} 6.0 \mathrm{~Hz}\right), 16.2\left(\mathrm{~d}, J_{\mathrm{PC}} 5.0\right.$ $\mathrm{Hz}), 50.0\left(\mathrm{~d}, J_{\mathrm{PC}} 155.0 \mathrm{~Hz}\right), 63.2\left(\mathrm{~d}, J_{\mathrm{PC}} 7.0 \mathrm{~Hz}\right), 63.4\left(\mathrm{~d}, J_{\mathrm{PC}} 7.0 \mathrm{~Hz}\right), 115.2\left(\mathrm{~d}, J_{\mathrm{CF}} 20.0 \mathrm{~Hz}\right)$, $129.6\left(\mathrm{~d}, J_{\mathrm{PC}} 6.0 \mathrm{~Hz}\right), 129.6-129.8(\mathrm{~m}), 132.2\left(\mathrm{~d}, J_{\mathrm{PC}} 3.0 \mathrm{~Hz}\right), 156.7,162.3\left(\mathrm{~d}, J_{\mathrm{FC}} 243 \mathrm{~Hz}\right) .{ }^{31} \mathrm{P}$ NMR $\left(\mathrm{CDCl}_{3} / \mathrm{H}_{3} \mathrm{PO}_{4}\right), \delta: 22.17$ (d, $J_{\mathrm{P}-\mathrm{F}} 3.2 \mathrm{~Hz}$ ). Anal. Calcd for $\mathrm{C}_{12} \mathrm{H}_{18} \mathrm{FN}_{2} \mathrm{O}_{4} \mathrm{P}$. C, 47.35; H, 5.96; N, 9.21. Found: C, 47.30; H, 5.73; N, 9.10.

Diethyl [a-ureido-(4-nitrophenyl)]methyl phosphonate (2f). Yellow solid, mp231-233 ${ }^{\circ} \mathrm{C} .{ }^{1} \mathrm{H}$ NMR $\left(\mathrm{CD}_{3} \mathrm{SOCD}_{3}, 400 \mathrm{MHz}\right), \delta: 1.12$ (t, J 7.2 Hz, 3H), 1.22 (t, J 7.2 Hz, 3H), 3.82-3.91 (m, 1H), 3.91-3.99 (m, 1H), 4.00-4.11 (m, 2H), 5.35 (dd, J 23.2 Hz, J 9.6 Hz, 1H), $5.87\left(\mathrm{~s}, \mathrm{NH}_{2}, 2 \mathrm{H}\right)$, 7.24 (br, NH 1H), 7.64 (d, J $8.8 \mathrm{~Hz}, 2 \mathrm{H}), 8.23$ (d, $J 8.4 \mathrm{~Hz}, 2 \mathrm{H}) .{ }^{13} \mathrm{C} \mathrm{NMR}\left(\mathrm{CD}_{3} \mathrm{SOCD}_{3}, 100.6\right.$ $\mathrm{MHz}), \delta: 16.5\left(\mathrm{~d}, J_{\mathrm{PC}} 5.0 \mathrm{~Hz}\right), 16.7$ (d, $\left.J_{\mathrm{PC}} 5.0 \mathrm{~Hz}\right), 50.6\left(\mathrm{~d}, J_{\mathrm{PC}} 150.0 \mathrm{~Hz}\right), 63.0\left(\mathrm{~d}, J_{\mathrm{PC}} 7.0 \mathrm{~Hz}\right)$, $63.3\left(\mathrm{~d}, J_{\mathrm{PC}} 7.0 \mathrm{~Hz}\right), 123.8\left(\mathrm{~d}, J_{\mathrm{PC}} 2.0 \mathrm{~Hz}\right), 129.4\left(\mathrm{~d}, J_{\mathrm{PC}} 5.0 \mathrm{~Hz}\right), 145.7,147.3\left(\mathrm{~d}, J_{\mathrm{PC}} 3.0 \mathrm{~Hz}\right)$, $157.9\left(\mathrm{~d}, J_{\mathrm{PC}} 10.0 \mathrm{~Hz}\right) .{ }^{31} \mathrm{P} \mathrm{NMR}\left(\mathrm{CD}_{3} \mathrm{SOCD}_{3} / \mathrm{H}_{3} \mathrm{PO}_{4}\right), \delta: 21.48$. Anal. Calcd for $\mathrm{C}_{12} \mathrm{H}_{18} \mathrm{~N}_{3} \mathrm{O}_{6} \mathrm{P}$. C, 43.49; H, 5.48; N, 12.69. Found: C, 43.76; H, 5.53; N, 12.51.

Diethyl [a-ureido-(3-flourophenyl)]methyl phosphonate (2g). White solid, mp200-202 ${ }^{\circ} \mathrm{C} .{ }^{1} \mathrm{H}$ NMR $\left(\mathrm{CDCl}_{3}, 400 \mathrm{MHz}\right), \delta: 1.07$ (t, $\left.J 6.8 \mathrm{~Hz}, 3 \mathrm{H}\right), 1.11$ (t, $\left.J 6.8 \mathrm{~Hz}, 3 \mathrm{H}\right), 3.76-3.90(\mathrm{~m}, 1 \mathrm{H})$, $3.91-4.12(\mathrm{~m}, 3 \mathrm{H}), 5.38(\mathrm{dd}, J 22.0 \mathrm{~Hz}, J 10.0 \mathrm{~Hz}, 1 \mathrm{H}), 6.95(\mathrm{t}, J 8.8 \mathrm{~Hz}, 2 \mathrm{H}), 7.35-7.45(\mathrm{~m}, 2 \mathrm{H}$ and $\mathrm{NH}) .{ }^{13} \mathrm{C} \mathrm{NMR}\left(\mathrm{CDCl}_{3}, 100.6 \mathrm{MHz}\right), \delta: 16.0\left(\mathrm{~d}, J_{\mathrm{PC}} 6.0 \mathrm{~Hz}\right), 16.2\left(\mathrm{~d}, J_{\mathrm{PC}} 6.0 \mathrm{~Hz}\right), 49.9(\mathrm{~d}$, $\left.J_{\mathrm{PC}} 156.0 \mathrm{~Hz}\right), 63.2\left(\mathrm{~d}, J_{\mathrm{PC}} 7.0 \mathrm{~Hz}\right), 63.5\left(\mathrm{~d}, J_{\mathrm{PC}} 7.0 \mathrm{~Hz}\right), 115.2\left(\mathrm{dd}, J_{\mathrm{CF}} 24.0 \mathrm{~Hz}, J_{\mathrm{PC}} 2.0 \mathrm{~Hz}\right)$, 129.6, 129.7 (d, $\left.J_{\mathrm{PC}} 2.0 \mathrm{~Hz}\right), 129.8,156.7$ (d, $\left.J_{\mathrm{PC}} 10.0 \mathrm{~Hz}\right), 162.3\left(\mathrm{dd}, J_{\mathrm{PC}} 3.0\right.$ and $\left.J_{\mathrm{FC}} 246 \mathrm{~Hz}\right)$. 
${ }^{31} \mathrm{P}$ NMR $\left(\mathrm{CDCl}_{3} / \mathrm{H}_{3} \mathrm{PO}_{4}\right), \delta: 22.17\left(\mathrm{~d}, J_{\mathrm{PF}} 3.2 \mathrm{~Hz}\right)$. Anal. Calcd for $\mathrm{C}_{12} \mathrm{H}_{18} \mathrm{FN}_{2} \mathrm{O}_{4} \mathrm{P} . \mathrm{C}, 47.35 ; \mathrm{H}$, 5.96; N, 9.21. Found: C, 47.21; H, 5.68; N, 9.20.

Diethyl [a-ureido-(2-chlorophenyl)]methyl phosphonate (2h). Yellow solid, mp194-196 ${ }^{\circ} \mathrm{C}$. ${ }^{1} \mathrm{H}$ NMR $\left(\mathrm{CD}_{3} \mathrm{SOCD}_{3}, 400 \mathrm{MHz}\right), \delta: 1.02$ (t, J 7.2 Hz, 3H), 1.25 (t, J 7.2 Hz, 3H), 3.63-3.74 (m, $1 \mathrm{H}), 3.78-3.90(\mathrm{~m}, 1 \mathrm{H}), 4.02-4.13(\mathrm{~m}, 2 \mathrm{H}), 5.69(\mathrm{dd}, J 22.0 \mathrm{~Hz}, J 10.0 \mathrm{~Hz}, 1 \mathrm{H}), 5.80\left(\mathrm{~s}, \mathrm{NH}_{2}\right.$, 2H), $7.28(\mathrm{dd}, J 10.0 \mathrm{~Hz}, J 4.0 \mathrm{~Hz}, 1 \mathrm{H}), 7.28-7.36(\mathrm{~m}, 1 \mathrm{H}), 7.37-7.49(\mathrm{~m}, 3 \mathrm{H}) .{ }^{13} \mathrm{C}$ NMR $\left(\mathrm{CD}_{3} \mathrm{SOCD}_{3}-\mathrm{TMS}, 100.6 \mathrm{MHz}\right), \delta: 16.4$ (d, $\left.J_{\mathrm{P}-\mathrm{C}} 5.0 \mathrm{~Hz}\right), 16.7$ (d, $\left.J_{\mathrm{P}-\mathrm{C}} 5.0 \mathrm{~Hz}\right), 47.2$ (d, $J_{\mathrm{P}-\mathrm{C}} 155.0$ $\mathrm{Hz}), 62.8\left(\mathrm{~d}, J_{\mathrm{P}-\mathrm{C}} 7.0 \mathrm{~Hz}\right), 63.1\left(\mathrm{~d}, J_{\mathrm{P}-\mathrm{C}} 7.0 \mathrm{~Hz}\right), 127.7$ (d, $\left.J_{\mathrm{P}-\mathrm{C}} 3.0 \mathrm{~Hz} \mathrm{~Hz}\right), 129.6,129.8\left(\mathrm{~d}, J_{\mathrm{P}-\mathrm{C}}\right.$ $3.0 \mathrm{~Hz}), \quad 133.2,133.3,133.8$ (d, $\left.J_{\mathrm{P}-\mathrm{C}} 1.0 \mathrm{~Hz}\right), 157.8$ (d, $\left.J_{\mathrm{P}-\mathrm{C}} 10.0 \mathrm{~Hz}\right) .{ }^{31} \mathrm{P} \quad \mathrm{NMR}$ $\left(\mathrm{CD}_{3} \mathrm{SOCD}_{3} / \mathrm{H}_{3} \mathrm{PO}_{4}\right)$, $\delta$ : 22.12. Anal. Calcd for $\mathrm{C}_{12} \mathrm{H}_{18} \mathrm{ClN}_{2} \mathrm{O}_{4} \mathrm{P}$. C, 44.99; H, 5.67; N, 8.75. Found: C, 45.11; H, 5.73; N, 8.68.

Diethyl [ $\alpha$-ureido-(1-naphthyl)]methyl phosphonate (2i). White solid, mp179-181 ${ }^{\circ} \mathrm{C} .{ }^{1} \mathrm{H}$ NMR $\left(\mathrm{CD}_{3} \mathrm{SOCD}_{3}, 400 \mathrm{MHz}\right), \delta: 0.82(\mathrm{t}, J 7.2 \mathrm{~Hz}, 3 \mathrm{H}), 1.26(\mathrm{t}, J 7.2 \mathrm{~Hz}, 3 \mathrm{H}), 3.47-3.64(\mathrm{~m}$, $1 \mathrm{H}), 3.72-3.83(\mathrm{~m}, 1 \mathrm{H}), 4.02-4.16(\mathrm{~m}, 2 \mathrm{H}), 5.8\left(\mathrm{~s}, 2 \mathrm{H}, \mathrm{NH}_{2}\right), 6.03(\mathrm{dd}, J 22.0 \mathrm{~Hz}, J 10.0 \mathrm{~Hz}, 1 \mathrm{H})$, 7.24 (dd, J $9.6 \mathrm{~Hz}, J 3.6 \mathrm{~Hz}, 1 \mathrm{H}), 7.52-7.63$ (m, 3H), 7.64-7.70 (m. 1H), 7.90 (d, J 8.4 Hz, 1H), $7.96(\mathrm{~d}, J 8.4 \mathrm{~Hz}, 1 \mathrm{H}), 8.20(\mathrm{~d}, J 8.4 \mathrm{~Hz}, 1 \mathrm{H}) .{ }^{13} \mathrm{C} \mathrm{NMR}\left(\mathrm{CD}_{3} \mathrm{SOCD}_{3}, 100.6 \mathrm{MHz}\right), \delta: 16.3(\mathrm{~d}$, $\left.J_{\mathrm{PC}} 5.0 \mathrm{~Hz}\right), 16.8\left(\mathrm{~d}, J_{\mathrm{PC}} 6.0 \mathrm{~Hz}\right), 46.1\left(\mathrm{~d}, J_{\mathrm{PC}} 152.0 \mathrm{~Hz}\right), 62.6\left(\mathrm{~d}, J_{\mathrm{PC}} 7.0 \mathrm{~Hz}\right), 63.0\left(\mathrm{~d}, J_{\mathrm{PC}} 7.0\right.$ $\mathrm{Hz}), 124.2,125.4\left(\mathrm{~d}, J_{\mathrm{PC}} 3.0 \mathrm{~Hz}\right), 126.2\left(\mathrm{~d}, J_{\mathrm{PC}} 4.0 \mathrm{~Hz}\right), 126.7,128.5$ (d, $\left.J_{\mathrm{PC}} 2.0 \mathrm{~Hz}\right), 129.0$, 131.3, 131.4, 133.7, 134.2, 158.0 (d, $\left.J_{\mathrm{PC}} 9.0 \mathrm{~Hz}\right) .{ }^{31} \mathrm{P} \mathrm{NMR}\left(\mathrm{CD}_{3} \mathrm{SOCD}_{3} / \mathrm{H}_{3} \mathrm{PO}_{4}\right), \delta$ : 23.13. Anal. Calcd for $\mathrm{C}_{16} \mathrm{H}_{21} \mathrm{~N}_{2} \mathrm{O}_{4} \mathrm{P}$. C, 57.12; H, 6.30; N, 8.33. Found: C, 57.11; H, 6.21; N, 8.30.

Diethyl [a-ureido-(2-naphthyl)]methylphosphonate (2j). White solid, mp178-179 ${ }^{\circ} \mathrm{C}$. ${ }^{1} \mathrm{H}$ NMR $\left(\mathrm{CD}_{3} \mathrm{SOCD}_{3}, 400 \mathrm{MHz}\right), \delta: 1.06(\mathrm{t}, J 7.2 \mathrm{~Hz}, 3 \mathrm{H}), 1.23$ (t, J 7.2 Hz, 3H), 3.70-3.83 (m, $1 \mathrm{H}), 3.84-3.96(\mathrm{~m}, 1 \mathrm{H}), 3.98-4.13(\mathrm{~m}, 2 \mathrm{H}), 5.34(\mathrm{dd}, J 22.0 \mathrm{~Hz}, J 10.0 \mathrm{~Hz}, 1 \mathrm{H}), 5.82\left(\mathrm{~s}, \mathrm{NH}_{2}\right.$, 2H), $7.53(\mathrm{dd}, J 10.0 \mathrm{~Hz}, J 4.0 \mathrm{~Hz}, 1 \mathrm{H}), 7.49-7.58(\mathrm{~m}, 3 \mathrm{H}), 7.86(\mathrm{~s}, 1 \mathrm{H}), 7.88-7.96(\mathrm{~m}, 3 \mathrm{H}) .{ }^{13} \mathrm{C}$ NMR (CD $\left.\mathrm{SOCD}_{3}, 100.6 \mathrm{MHz}\right), \delta: 16.4\left(\mathrm{~d}, J_{\mathrm{PC}} 6.0 \mathrm{~Hz}\right), 16.7\left(\mathrm{~d}, J_{\mathrm{PC}} 5.0 \mathrm{~Hz}\right), 50.6\left(\mathrm{~d}, J_{\mathrm{PC}} 153.0\right.$ $\mathrm{Hz}), 63.0\left(\mathrm{~d}, J_{\mathrm{PC}} 7.0 \mathrm{~Hz}\right), 63.3\left(\mathrm{~d}, J_{\mathrm{PC}} 7.0 \mathrm{~Hz}\right), 126.3\left(\mathrm{~d}, J_{\mathrm{PC}} 4.0 \mathrm{~Hz}\right), 126.7,126.8$ (d, $\left.J_{\mathrm{PC}} 6.0 \mathrm{~Hz}\right)$, 126.9, 128.0, 128.1, 128.2, 132.7, 133.0 (d, $\left.J_{\mathrm{PC}} 2.0 \mathrm{~Hz}\right), 134.8,158.2$ (d, $\left.J_{\mathrm{PC}} 10.0 \mathrm{~Hz}\right) .{ }^{31} \mathrm{P}$ NMR $\left(\mathrm{CD}_{3} \mathrm{SOCD}_{3} / \mathrm{H}_{3} \mathrm{PO}_{4}\right)$, $\delta: 22.87$. Anal. Calcd for $\mathrm{C}_{16} \mathrm{H}_{21} \mathrm{~N}_{2} \mathrm{O}_{4} \mathrm{P}$. C, 57.12; H, 6.30; N, 8.33. Found: C, 56.95; H, 6.20; N, 8.25.

Tetrakisethyl ( $\alpha$-urylene diphenylmethyl) diphosphonate (3k). White solid, ${ }^{1} \mathrm{H} \mathrm{NMR}\left(\mathrm{CDCl}_{3}\right.$, $400 \mathrm{MHz}$ ), $\delta: 1.04-1.14(\mathrm{~m}, 9 \mathrm{H}), 1.33$ (t, J 7.2 Hz, 3H), 3.70-3.83 (m, 2H), 3.84-3.96 (m, 4H), 3.98-4.19 (m, 2H), $5.45(\mathrm{dd}, J 22.0 \mathrm{~Hz}, J 10.0 \mathrm{~Hz}, 2 \mathrm{H}$, simplified from doublet of doublet to doublet by addition of $\left.\mathrm{D}_{2} \mathrm{O}\right), 7.24-7.33(\mathrm{~m}, 6 \mathrm{H}), 7.35-7.50(\mathrm{~m}, 4 \mathrm{H}$ and $2 \mathrm{NH}) .{ }^{13} \mathrm{C} \mathrm{NMR}\left(\mathrm{CDCl}_{3}\right.$,

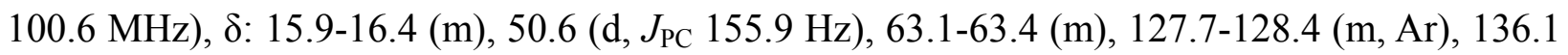
$\left(\mathrm{d}, J_{\mathrm{PC}} 2.5 \mathrm{~Hz}\right), 158.2\left(\mathrm{~d}, J_{\mathrm{PC}} 10 \mathrm{~Hz}\right) .{ }^{31} \mathrm{P} \mathrm{NMR}\left(\mathrm{CDCl}_{3} / \mathrm{H}_{3} \mathrm{PO}_{4}\right), \delta: 22.53$ and 22.76. HRMS calcd for $\mathrm{C}_{18} \mathrm{H}_{34} \mathrm{O}_{7} \mathrm{~N}_{2} \mathrm{P}_{2}\left(\mathrm{MNa}^{+}\right)$: 513.1706. Found 513.1916.

Tetrakisethyl ( $\alpha$-urylene dithiophenylmethyl) diphosphonate (3I). White solid. ${ }^{1} \mathrm{H}$ NMR $\left(\mathrm{CDCl}_{3}, 400 \mathrm{MHz}\right), \delta: 1.08-1.17(\mathrm{~m}, 9 \mathrm{H}), 1.24(\mathrm{t}, J 7.2 \mathrm{~Hz}, 3 \mathrm{H}), 3.80-4.15(\mathrm{~m}, 8 \mathrm{H}), 5.45(\mathrm{dd}, J$ $22.0 \mathrm{~Hz}, J 10.0 \mathrm{~Hz}, 2 \mathrm{H}$, simplified from doublet of doublet to doublet by addition of $\left.\mathrm{D}_{2} \mathrm{O}\right), 6.90$ $7.10(\mathrm{~m}, 4 \mathrm{H}), 7.20-7.30(\mathrm{~m}, 2 \mathrm{NH}), 7.40-7.55$ (m, 2H). ${ }^{13} \mathrm{C} \mathrm{NMR}\left(\mathrm{CDCl}_{3}, 100.6 \mathrm{MHz}\right), \delta: 16.4-$ 
$16.6(\mathrm{~m}), 46.4\left(\mathrm{~d}, J_{\mathrm{PC}} 160.0 \mathrm{~Hz}\right), 46.5\left(\mathrm{~d}, J_{\mathrm{PC}} 160.0 \mathrm{~Hz}\right)$ 63.4-63.7 (m), 126.3-127.6 (m, Ar), $139.2\left(\mathrm{~d}, J_{\mathrm{PC}} 4.0 \mathrm{~Hz}\right), 156.2\left(\mathrm{~d}, J_{\mathrm{PC}} 11 \mathrm{~Hz}\right) .{ }^{31} \mathrm{P} \mathrm{NMR}\left(\mathrm{CDCl}_{3} / \mathrm{H}_{3} \mathrm{PO}_{4}\right), \delta: 20.46$ and 20.64. Anal. Calcd for $\mathrm{C}_{19} \mathrm{H}_{30} \mathrm{~N}_{2} \mathrm{O}_{7} \mathrm{PS}_{2}$. C, 43.50; H, 5.77; N, 5.34. Found: C, 43.40; H, 5.38; N, 5.25.

Diethyl [a-thioureido-(4-chlorophenyl)]methyl phosphonate (4a). Yellow solid, mp180-182 ${ }^{\circ} \mathrm{C}$. ${ }^{1} \mathrm{H}$ NMR $\left(\mathrm{CDCl}_{3}, 400 \mathrm{MHz}\right), \delta: 1.15(\mathrm{t}, J 7.2 \mathrm{~Hz}, 3 \mathrm{H}), 1.41$ (t, J 7.2 Hz, 3H), 3.71-3.83 (m, 1H), 3.87-3.99 (m, 1H), 4.17-4.35 (m, 2H), 6.25 (dd, J $21.2 \mathrm{~Hz}, J 9.6 \mathrm{~Hz}, 1 \mathrm{H}), 6.62\left(\mathrm{br}, \mathrm{NH}_{2}\right.$, 2H), $7.33(\mathrm{~d}, J 8.4 \mathrm{~Hz}, 2 \mathrm{H}), 7.44(\mathrm{~d}, J 8.4 \mathrm{~Hz}, 1 \mathrm{H}), 8.93(\mathrm{~d}, J 6.8 \mathrm{~Hz}, 1 \mathrm{H}, \mathrm{NH}) .{ }^{13} \mathrm{C}$ NMR $\left(\mathrm{CDCl}_{3}, 100.6 \mathrm{MHz}\right), \delta: 16.1\left(\mathrm{~d}, J_{\mathrm{PC}} 6.0 \mathrm{~Hz}\right), 16.5\left(\mathrm{~d}, J_{\mathrm{PC}} 6.0 \mathrm{~Hz}\right), 54.6\left(\mathrm{~d}, J_{\mathrm{PC}} 154.0 \mathrm{~Hz}\right), 63.9(\mathrm{~d}$, $\left.J_{\mathrm{PC}} 6.0 \mathrm{~Hz}\right), 64.4\left(\mathrm{~d}, J_{\mathrm{PC}} 7.0 \mathrm{~Hz}\right), 128.8\left(\mathrm{~d}, J_{\mathrm{PC}} 2.0 \mathrm{~Hz} \mathrm{~Hz}\right), 129.7$ (d, $\left.J_{\mathrm{PC}} 6.0 \mathrm{~Hz}\right), 133.4,134.3$ (d, $\left.J_{\mathrm{PC}} 3.0 \mathrm{~Hz}\right), 184.5\left(\mathrm{~d}, J_{\mathrm{PC}} 12.0 \mathrm{~Hz}\right) .{ }^{31} \mathrm{P} \mathrm{NMR}\left(\mathrm{CDCl}_{3} / \mathrm{H}_{3} \mathrm{PO}_{4}\right), \delta$ : 21.25. Anal. Calcd for $\mathrm{C}_{12} \mathrm{H}_{18} \mathrm{~N}_{2} \mathrm{ClO}_{3}$ PS. C, 42.85; H, 5.40; N, 8.33. Found: C, 42.91; H, 5.20; N, 8.21.

\section{Acknowledgements}

The authors gratefully acknowledge support by the Institute for Advanced Studies in Basic Sciences (IASBS) Research Council under grant No. G2010IASBS120. The authors thank to Mr. Haruhiko Fukaya, Tokyo University of Pharmacy and Life Sciences for their helps carrying out the X-ray crystallographic analysis.

\section{References}

1. (a) Engel, R. Chem Rev. 1977, 77, 349. (b) Hiratake, J.; Oda, J. Biosci. Biotechnol. Biochem. 1997, 61, 211. (c) Schug, K. A.; Lindner. W. Chem. Rev. 2005, 105, 67. (d) Moonen, K.; Laureyn, I.; Stevens, C. V. Chem. Rev. 2004, 104, 6177. (e) Palacios, F.; Alonso, C.; de los Santos, J. M. Current Organic Chemistry 2004, 8, 1481.

2. Collinsova, M.; Jiracek, J. Curr. Med. Chem. 2000, 7, 629.

3. Kafarski, P.; Lejczak, B.; Tyka, R.; Koba, L.; Pliszczak, E.; Wieczorek, P. J. Plant Growth Regul. 1995, 14, 199.

4. Miliszkiewicz, D.; Wieczorek, P.; Lejcezak, B.; Kowalik, E. Kafarski, P. Pesti. Sci. 1992, $34,349$.

5. Ishiguri, Y.; Yamada, Y.; Kato, T.; Sasaki, M.; Mukai, K. Eur. Pat. Appl., EP 82-301905, 1982; Chem. Abstr. 1983, 98, 102686.

6. Kukhar, V. P.; Hudson, H. R. In Aminophosphonic and Aminophosphinic Acids, Wiley: Chichester, 2000.

7. (a) Gancarz, R.; Chakraborty, S. Synthesis 1977, 625. (b) Giannousis, P. P.; Bartlett, P. A. J. Med. Chem. 1987, 30, 1603. (c) Maier, L.; Lea, P. J. Phosphorus, Sulfur, Silicon 1983, 17, 1. (d) Hilderbrand, R. L. In The Role of Phosphonates in Living Systems, CRC Press: Boca 
Raton, FL, 1982. (e) Kafarski, P.; Lejczak, B. Phosphorus, Sulfur, Silicon 1991, 63, 193215. (f) Hanessian, S.; Bennani, Y. L. Synthesis 1995, 1272.

8. (a) Redmore, D. In Topics in Phosphorus Chemistry; Griffith, E. J.; Grayson, M. Eds.; Vol. 8; Wiley: New York, 1976. (b) Atherton, F. R.; Hassall, C. H.; Lambert, R. W. J. Med. Chem. 1986, 29, 29. (c) Allen, M. C.; Fuhrer, W.; Tuck, B.; Wade, R.; Wood, J. M. J. Med. Chem. 1989, 32, 1652. (d) Hassall, C. H. In Antibiotics; Hahn, F. E. Ed., Springer Verlag: Berlin, 1983, Vol VI, 1-11. (e) Gancarz, R.; Wieczorek, J. S. Synthesis 1978, 625. (f) Seyferth, D.; Marmor, R. S.; Hilbert, P. J. Org. Chem. 1971, 36, 1379. (g) Worms, K. H.; Schmidt-Dunker, M. In Organic Phosphorus Compounds, Kosolapoff, G. M.; Marier, L., Eds., Wiley: New York, 1976; Vol. 7, p 1. (h) Kaboudin, B. Phosphorus, Sulfur, Silicon 2002, 177, 1749. (i) Bhagat, S.; Chakraborti, A. K. J. Org. Chem. 2007, 72, 1263.

9. (a) Barycki, J., Mastalerz, P.; Soroka, M. Tetrahedron Lett. 1970, 36, 3147. (b) Kaboudin, B.; Sorbiun, M. Tetrahedron Lett. 2007, 48, 9015.

10. Huber III, J. W.; Gilmore, W. F. Tetrahedron Lett. 1979, 33, 30498.

11. Hudson, H. R.; Ismail, F.; Pianka, M. Phosphorus, Sulfur and Silicon 2001, 173, 143.

12. Chen, M. H.; Chen, Z.; Song, B. A.; Bhadury, P. S.; Yang, S.; Cai, X. J.; Hu, D. Y.; Xue, W.; Zeng, S. J. Agric. Food Chem. 2009, 57, 1383.

13. Zhu, Y.; Sun, C.; Wu, W. J. Univ.Sci. Tecnol. Bejing 2007, 14, 1.

14. Meyer, W.; Bohner, B.; Dawes, D. U.S. Patent Number US 3957 924, 1976.

15. Review: Ordonez, M.; Rajas-Cabrera, H.; Cativiela, C. Tetrahedron 2009, 65, 17.

16. (a) Birum, G. H. J. Org. Chem. 1974, 39, 209. (b) Huber III, J. W.; Gilmore, W. F. Tetrahedron Lett. 1979, 33, 3049.

17. Palacios, F.; Ochoa de Retana, A. M.; Oyarzabal, J. Tetrahedron 1999, 55, 3091.

18. For example: (a) Kaboudin, B. Chem. Lett. 2001, 880. (b) Kaboudin, B.; Nazari, R. Synth. Commun. 2001, 31, 2245. (c) Kaboudin, B.; Nazari, R. Tetrahedron Lett. 2001, 42, 8211. (d) Kaboudin, B.; Rahmani, A. Synthesis 2003, 2705. (e) Kaboudin, B.; Saadati, F. Synthesis 2004, 1249. (f) Kaboudin, B.; Rahmani, A. Org. Prep. Proced. Int. 2004, 36, 82. (g) Kaboudin B.; Moradi, K. Tetrahedron Lett. 2005, 46, 2989. (h) Kaboudin, B.; Haghighat, H. Tetrahedron Lett. 2005, 46, 7955. (i) Kaboudin, B.; Karimi, M. Bioorg. Med. Chem. Lett. 2006, 16, 5324. (j) Kaboudin, B.; Moradi, K. Synthesis 2006, 2339. (k) Kaboudin, B.; Jafari, E. Synthesis 2006, 3063. (1) Kaboudin, B.; Farjadian, F. Beilstein J. Org. Chem. 2006, 2:4. (m) Kaboudin, B.; Alipour, S. Tetrahedron Lett. 2009, 50, 4243. (n) Kaboudin, B.; Saadati, F. Tetrahedron Lett. 2009, 50, 1450. 